\title{
Renal double negative T cells: unconventional cells in search of a function
}

\author{
Raphael A. Nemenoff ${ }^{1,2}$, Emily K. Kleczko ${ }^{1}$, Katharina Hopp ${ }^{1,2}$ \\ ${ }^{1}$ Department of Medicine, Division of Renal Diseases and Hypertension, ${ }^{2}$ Consortium for Fibrosis Research and Translation, University of Colorado, \\ Denver, Anschutz Medical Campus, Aurora, CO, USA \\ Correspondence to: Dr. Katharina Hopp, PhD. Division of Renal Diseases and Hypertension, PKD Program, University of Colorado, Denver, \\ Anschutz Medical Campus, 12700 E 19th Avenue, P15-7400A, Mail Stop C281, Aurora, CO 80045, USA. Email: Katharina.hopp@cuanschutz.edu. \\ Provenance: This is an article commissioned by the Section Editor Dr. Cheng Yuan (Zhongnan Hospital, Wuhan University, Wuhan, China). \\ Comment on: Sadasivam M, Noel S, Lee SA. Activation and Proliferation of PD- $1^{+}$Kidney Double-Negative T Cells Is Dependent on Nonclassical \\ MHC Proteins and IL-2. J Am Soc Nephrol 2019;30:277-92.
}

Submitted Sep 06, 2019. Accepted for publication Sep 18, 2019.

doi: 10.21037/atm.2019.09.107

View this article at: http://dx.doi.org/10.21037/atm.2019.09.107

In studying the role of adaptive immunity in disease, investigators have focused extensively on $\mathrm{CD} 8^{+} \mathrm{T}$ cells (effector $\mathrm{T}$ cells) and $\mathrm{CD}^{+}{ }^{+} \mathrm{T}$ cells (helper $\mathrm{T}$ cells). For many years, double negative (DN) $\mathrm{T}$ cells $\left(\mathrm{CD} 4^{-} \mathrm{CD} 8^{-}\right)$ have been neglected by immunologists due to their overall rarity and lack of clear expression markers, making them difficult to study. However, within the last decade, these unconventional $\mathrm{T}$ cells have been found to be important in various disease presentations, resulting in increased interest in this population as a therapeutic target. Interestingly, DN T cells have been found to be both pro- and antiinflammatory (1). Several studies hint towards DN T cells being key regulators of autoimmunity. For example, in Systemic Lupus Erythematosus (SLE) patients, DN T cell numbers were found to be significantly increased and were a major producer of IL-17, the key inflammatory cytokine in SLE (2). Similarly, in Autoimmune Lymphoproliferative Syndrome (ALPS) patients, peripheral DN T cell numbers have been reported to increase from $1 \%$ in controls to $40 \%$ of $\mathrm{T}$ cells, making DN $\mathrm{T}$ cell number a potential disease biomarker (3). Peripheral DN T cell numbers have also been shown to increase in HIV patients. Interestingly, these numbers decrease upon successful antiviral therapy, suggesting they may contribute to viral production and are sensitive to active antiretroviral therapy (4). Furthermore, DN T cells have been found to be the major responders to Francisella tularensis (5) and Listeria monocytogenes (6) infections. Contrary to their proinflammatory role, DN
$\mathrm{T}$ cells have been proposed to be essential in maintenance of immune homeostasis and self-tolerance. In that respect, several studies have shown that DN T cells play an important role in the development of tolerance after transplantation (7). Additionally, DN T cells have been proposed to provide long lasting protection against type-I diabetes in diabetes-prone NOD mice (8).

Remarkably, while relatively rare in totality in humans and mice alike (1-5\% of all $\mathrm{T}$ cells) $(1,9), \mathrm{DN} \mathrm{T}$ cells seem to preferentially reside in specific organs/tissues, suggesting they may have specific functions in these settings. For example, it has been reported that DN T cells accumulate in the liver of several autoimmuneprone mouse strains, with their numbers increasing as mice become older and more diseased (10). Further, DN T cells have been found to be the dominant $\mathrm{T}$ cell population in both the intestine and the female genital track $(11,12)$. In the kidney, increased numbers of DN $\mathrm{T}$ cells were first demonstrated by Ascon et al. in 2008, who found that DN T cells comprise $\sim 23 \%$ of all renal $\mathrm{T}$ cells $\left(\mathrm{CD}^{+} 55 \%, \mathrm{CD} 8^{+} 21 \%\right)$ in 8 -week-old wildtype C57Bl/6 mice (13). Similarly, Martina et al. found that the DN T cell frequency in normal human kidney can range between $18-61 \%$ of all renal $\mathrm{T}$ cells (14). However, other investigators have found different levels of this population. Zimmerman et al. reported that only $\sim 5 \%$ of all renal $\mathrm{T}$ cells are DN T cells in normal human kidney (15), and on the mouse side, renal DN T cells in 8-week- 
A
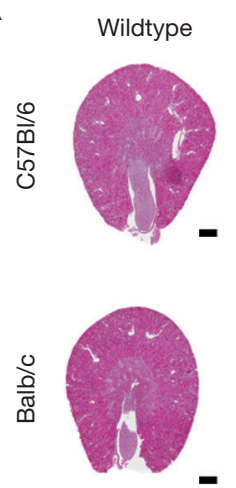
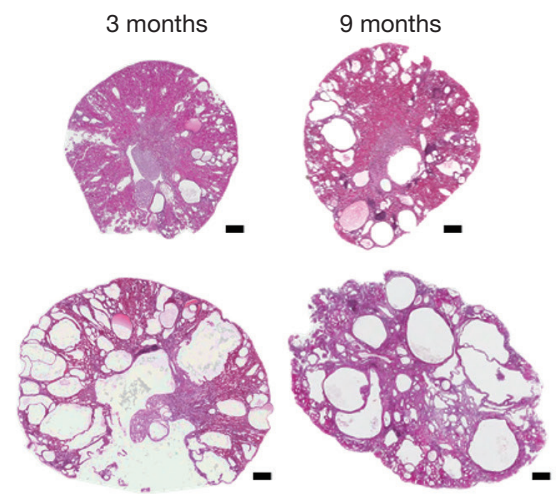

B $\quad \mathrm{C} 57 \mathrm{~B} / / 6$

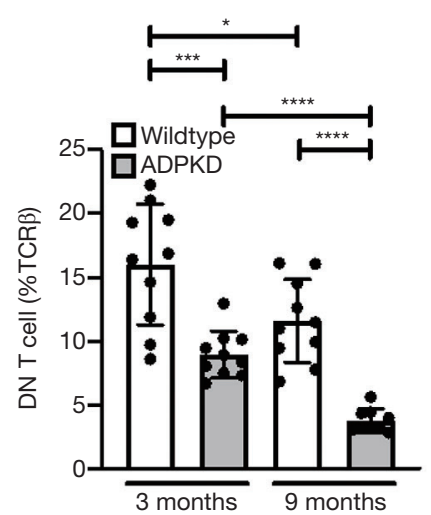

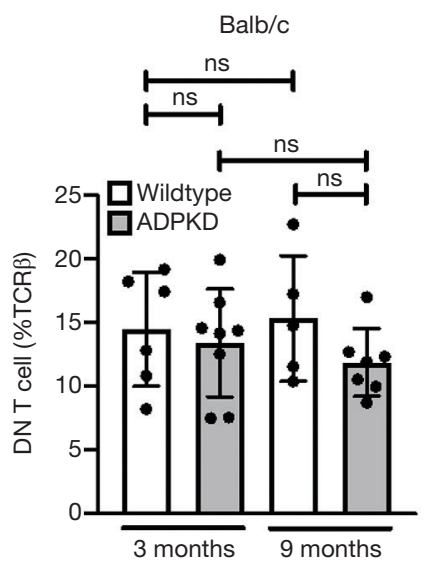

C

\begin{tabular}{|c|c|c|c|c|c|c|c|c|c|c|c|c|}
\hline \multirow{3}{*}{ T cell population } & \multicolumn{6}{|c|}{$\mathrm{C} 57 \mathrm{BI} / 6$} & \multicolumn{6}{|c|}{ Balb/c } \\
\hline & \multicolumn{3}{|c|}{3 months } & \multicolumn{3}{|c|}{9 months } & \multicolumn{3}{|c|}{3 months } & \multicolumn{3}{|c|}{9 months } \\
\hline & Wildtype & ADPKD & $P$ value & Wildtype & ADPKD & $P$ value & Wildtype & ADPKD & $P$ value & Wildtype & ADPKD & $P$ value \\
\hline DN T cells (\%live) & $0.047 \pm 0.038$ & $0.059 \pm 0.031$ & 0.46 & $0.037 \pm 0.012$ & $0.10 \pm 0.074$ & 0.013 & $0.018 \pm 0.0039$ & $0.12 \pm 0.035$ & $<0.0001$ & $0.039 \pm 0.031$ & $0.37 \pm 0.091$ & 0.0001 \\
\hline DN T cells $(\%$ TCR $\beta)$ & $16.01 \pm 4.73$ & $8.95 \pm 1.83$ & 0.0003 & $11.58 \pm 3.24$ & $3.77 \pm 0.90$ & $<0.0001$ & $14.43 \pm 4.47$ & $13.37 \pm 4.23$ & 0.66 & $15.30 \pm 4.92$ & $11.85 \pm 2.66$ & 0.15 \\
\hline $\mathrm{CD}^{+} \mathrm{T}$ cells $(\% \mathrm{TCR} \beta)$ & $39.55 \pm 5.32$ & $46.54 \pm 6.74$ & 0.019 & $38.07 \pm 8.44$ & $48.37 \pm 5.10$ & 0.0039 & $63.51 \pm 1.76$ & $61.70 \pm 5.99$ & 0.49 & $48.12 \pm 7.73$ & $57.09 \pm 5.09$ & 0.035 \\
\hline $\mathrm{CD}^{+} \mathrm{T}$ cells $(\% \mathrm{TCR} \beta)$ & $41.27 \pm 7.83$ & $42.38 \pm 6.70$ & 0.74 & $45.77 \pm 9.15$ & $43.89 \pm 4.50$ & 0.57 & $19.20 \pm 4.84$ & $20.95 \pm 3.06$ & 0.42 & $31.68 \pm 10.27$ & $27.56 \pm 4.96$ & 0.37 \\
\hline $\mathrm{DN}, \mathrm{Pd}-1^{+} \mathrm{T}$ cells $(\% \mathrm{TCR} \beta)$ & $\mathrm{N} / \mathrm{A}$ & N/A & N/A & $3.25 \pm 1.05$ & $7.79 \pm 4.80$ & 0.0092 & N/A & N/A & N/A & $2.09 \pm 1.84$ & $6.93 \pm 3.74$ & 0.025 \\
\hline
\end{tabular}

Figure 1 Renal DN T cell frequency decreases with age and ADPKD severity in the C57Bl/6 background. (A) H\&E stained renal cross sections of wildtype and ADPKD mice. In the orthologous ADPKD model, $P k d 1^{\mathrm{R} 3277 \mathrm{C} / R 3277 \mathrm{C}}(17,18)$, polycystic kidney disease severity increases with age and overall disease severity is more pronounced in the Balb/c versus C57Bl/6 strain; (B) renal frequency of DN T cells as percent of all renal $\alpha \beta \mathrm{T}$ cells. DN T cells were gated using $\mathrm{CD}^{2} 5^{+}, \mathrm{TCR} \beta^{+}, \mathrm{MHC} \mathrm{II}^{-}, \mathrm{CD}^{-}, \mathrm{CD}^{-}$. Note, this gating strategy does not differentiate between "true" DN T cells and NKT cells. In the C57Bl/6 strain, renal DN T cell frequency (\% TCR $\beta$ ) is significantly lower in ADPKD mice compared to wildtype mice and decreases significantly with age in both wildtype and diseased animals. In Balb/c mice, renal DN T cell frequencies remain steady independent of disease state or age; (C) tabular summary of renal T cell distribution. Globally, renal DN T cell numbers significantly increase with ADPKD severity in both strains (\%live), which correlates to an overall increase in all renal T cell subpopulations as published previously (17). Renal DN T cells expressing Pd-1 are rare using our mouse perfusion and kidney digestion protocol as published previously (17), but this rare population is nonetheless significantly elevated in ADPKD mice compared to control. Scale bar $500 \mu \mathrm{m}$, statistics are expressed as mean $\pm \mathrm{SD}$, Statistical comparisons were made using unpaired $t$-test with Welch correction, *, $\mathrm{P}<0.05 ;{ }^{* *}, \mathrm{P}<0.01 ;{ }^{* * *}, \mathrm{P}<0.001$; **** $\mathrm{P}<0.0001$. DN, double negative; ADPKD, autosomal dominant polycystic kidney disease.

old wildtype $\mathrm{C} 57 \mathrm{Bl} / 6$ mice have been reported to be either $\sim 28 \%$ (16) or $\sim 35 \%$ (14) of the total renal $\mathrm{T}$ cell population. While these discrepancies may be in part due to technical difficulties associated with studying DN T cells, which will be discussed later, it is likely that DN T cell frequency can change dependent on the age and strain of the mouse. For example, our laboratory has found renal DN $\mathrm{T}$ cell frequency to decrease with age in wildtype C57Bl/6 mice (3-month of age: 16\%; 9-month of age: $\sim 12 \% ; \mathrm{P}<0.05$ ); however, their frequency stayed steady throughout aging in wildtype Balb/C mice (14-15\%).

The role of DN T cells in renal disease, and how these populations are altered has not been extensively studied to date. In the case of SLE, Crispin et al was the first to report that SLE patients have a significant number of DN T cells infiltrating the kidney compared to controls (2). Similarly, Zimmerman et al. showed that DN T cell numbers increase in human Autosomal Dominant Polycystic Kidney Disease (ADPKD) kidneys compared to controls; however, this correlated with an overall renal $\mathrm{T}$ cell number increase (15). Here, the percentage of DN T cells as percent of total renal $\mathrm{T}$ cells was only marginally increased in ADPKD patients compared to controls (control: 5\%; ADPKD: 8\%) (15). In our mouse model of ADPKD (Figure $1 A)(17,18)$, we 
also found that the overall number of renal DN $\mathrm{T}$ cells increased with disease severity independent of strain, however, their frequency as percentage of total renal $\mathrm{T}$ cells decreased compared to wildtype and as disease advanced in the $\mathrm{C} 57 \mathrm{Bl} / 6$ strain but remained constant in the Balb/ $\mathrm{C}$ strain (Figure 1B,C). In acute kidney injury (AKI), DN $\mathrm{T}$ cell frequency has been examined in two separate papers using the same murine ischemia-reperfusion injury (IRI) model. Interestingly, Ascon et al. found that DN T cell numbers decrease $24 \mathrm{~h}$ after ischemic insult (13), while Martina et al found that both the total number as well as the percent of DN T cells as percent of total renal T cells increase $24 \mathrm{~h}$ after ischemic insult and decrease $72 \mathrm{~h}$ post insult compared to sham operated mice (14). The latter publication would suggest DN T cells are innate-like, early responders of IRI, and the group went on to show that DN T cells are a major producer of the anti-inflammatory cytokine IL-10 in steady state and early AKI (14). Further, in the same manuscript, Martina et al. also showed that adoptive transfer of DN $\mathrm{T}$ cells protected wildtype mice against AKI.

In their most recent paper published in $7 \mathrm{Am} \mathrm{Soc}$ Nephrol [Sadasivam et al. (16)], the same group that initially reported a functional role of renal DN T cells in AKI, defined mechanisms of renal DN T cell homeostasis. In general, mechanisms of DN T cell origin and homeostasis are incompletely understood, and literature addressing these concepts for renal DN T cells is lacking $(1,19)$. Using $\mathrm{B} 2 \mathrm{~m}^{\mathrm{tm} 1 \mathrm{Unc}}$ (MHC Ia and Ib null), H2 ${ }^{\text {dlAbl-Ea }}$ (MHC II null), and $\mathrm{H} 2 \mathrm{~K}^{\mathrm{b}} \mathrm{D}^{\mathrm{b}}$ (MHC Ia null) mice, they presented data showing that renal DN T cell homeostasis is primarily regulated by non-classical MHC Ib molecules, as B2 $\mathrm{m}^{\text {tmIUnc }}$ mice showed a significant reduction of renal DN T cells from $30 \%$ to $10 \%$, while renal DN T cell numbers were not impacted in $\mathrm{H} 2^{\mathrm{dlAb} 1-\mathrm{Ea}}$ and $\mathrm{H} 2 \mathrm{~K}^{\mathrm{b}} \mathrm{D}^{\mathrm{b}}$ mice. The group also showed that wildtype murine and normal human DN T cells express two distinct markers, $\mathrm{PD}-1^{+}(33 \%$ of $\mathrm{DN}$ $\mathrm{T}$ cells) and NK1.1 (30\% of DN T cells). Interestingly, in $\mathrm{B} 2 \mathrm{~m}^{\mathrm{tm} 1 \mathrm{Unc}}$ mice, the distribution of renal DN T cells expressing $\mathrm{Nk} 1.1^{+}$or $\mathrm{Pd}-1^{+}$changed in favor of $\mathrm{Pd}-1^{+}$ expressing cells; nearly no $\mathrm{Nk} 1.1^{+}$positive $\mathrm{DN} \mathrm{T}$ cells were present in the kidneys of $\mathrm{B} 2 \mathrm{~m}^{\mathrm{tm} 1 \mathrm{Unc}}$ mice. The authors also showed that in $\mathrm{B} 2 \mathrm{~m}^{\mathrm{tm} 1 \mathrm{Unc}}$ mice, overall $\mathrm{DN}$ $\mathrm{T}$ cell activation decreased by $15 \%\left(\mathrm{CD} 69^{+} \mathrm{DN} \mathrm{T}\right.$ cells), proliferation decreased by $30 \%$ (Ki67 ${ }^{+} \mathrm{DN} \mathrm{T}$ cells), and apoptosis increased by $8 \%$ (Annexin $\mathrm{V}^{+} \mathrm{DN} \mathrm{T}$ cells). Taken together the authors suggested that the reduction of renal DN T cells in B2 $\mathrm{m}^{\text {tmlUnc }}$ mice is due to loss of the
$\mathrm{Nk} 1.1^{+}$population and impaired proliferation/increased apoptosis of the $\mathrm{Pd}-1^{+}$population. However, the authors failed to experimentally prove this. It is possible that the only population impacted by MHC I loss is the Nk1.1 population. One caveat of the $\mathrm{B} 2 \mathrm{~m}^{\text {tmlUnc }}$ mice is that not only antigen presentation through MHC I is lost, but also all $\mathrm{CD}^{+} \mathrm{T}$ cells are lost. To better delineate whether MHC I molecules or $\mathrm{CD} 8^{+} \mathrm{T}$ cells are important for DN T cell homeostasis, proliferation, and activation, the authors adoptively transferred $\mathrm{CD} 8^{+} \mathrm{T}$ cells into $\mathrm{B} 2 \mathrm{~m}^{\mathrm{tm} 1 \mathrm{Unc}}$ mice. This restored DN T cell numbers and activation to $\sim 65 \%$ of wildtype levels. Since, transferred $\mathrm{CD}^{+} \mathrm{T}$ cells carry endogenous MHC I, they also adoptively transferred $\mathrm{CD}^{+}{ }^{+} \mathrm{T}$ cells or $\mathrm{B}$ cells alone as these cell types do not express MHC I. Surprisingly, adoptive transfer of CD4 ${ }^{+} \mathrm{T}$ cells, which are endogenously already present in $\mathrm{B} 2 \mathrm{~m}^{\text {tm1 Unc }}$ mice, restored DN T cell numbers to $48 \%$ and activation to $\sim 18 \%$ of wildtype levels. Adoptive B cell transfer had no impact on renal DN T cell numbers.

To understand what $T$ cell derived signal may stimulate $\mathrm{DN} T$ cell expansion, the authors moved to an in vitro co-culture system of DN and $\mathrm{CD} 8^{+} \mathrm{T}$ cells and assayed the secretion of key regulatory cytokines. They found significant increases in secreted IL-2, IL-17, INF- $\gamma$, and TNF- $\alpha$, but not IL-4, IL-6, or IL-10. The authors chose to focus on IL-2 as a key regulator of DN T cell expansion, as in vitro blockade of IL-2 hampered DN T cell expansion; blockade of the other cytokines was not tested. Interestingly, the in vivo support for their conclusion that IL-2 is necessary for DN T cell expansion was underwhelming - in B2 $\mathrm{m}^{\text {tm } 1 \mathrm{Unc}}$ mice adoptively transferred with CD8 ${ }^{+} \mathrm{T}$ cells, IL-2 levels rose 1.5 -fold, which was not significant and only $~ 30 \%$ of wildtype levels. Further, recombinant IL-2 given to $\mathrm{B} 2 \mathrm{~m}^{\text {tmlunc }}$ only restored DN $\mathrm{T}$ cells to $\sim 25 \%$ of wildtype levels, the exact levels were difficult to assess precisely as a wildtype group was missing in the experimental design. Also, the functional impact $\mathrm{CD}^{+} \mathrm{T}$ cells have on DN T cell expansion was not further evaluated. One last key observation the authors stated is that $24 \mathrm{~h}$ post IRI, only the $\mathrm{Pd}-1^{+} \mathrm{DN} \mathrm{T}$ cell population expanded while the $\mathrm{Nk} 1.1^{+}$population decreased, suggesting that $\mathrm{Pd}-1^{+} \mathrm{DN}$ T cells are the first responders of AKI. Interestingly, $\mathrm{B} 2 \mathrm{~m}^{\text {tmlUnc }}$ mice which lack $\mathrm{CD}^{+} \mathrm{T}$ cells and Nk1.1 ${ }^{+}$DN T cells showed similar severity of AKI 24 $h$ post IRI, suggesting that these populations are irrelevant to early AKI pathogenesis. This observation is in line with previously published studies which showed that $\mathrm{CD}^{+}$but not $\mathrm{CD} 8^{+} \mathrm{T}$ cells aggravate AKI $(20,21)$. However, the 
literature is inconsistent in this aspect, as $\mathrm{CD}^{+} \mathrm{T}$ cells have also been found to be either detrimental or protective to acute renal failure depending on the AKI model $(22,23)$.

While the study from Sadasivam et al. (16) provides new insight into the homeostasis of renal DN T cells, many questions remain regarding the role of these unconventional $\mathrm{T}$ cells in the kidney, especially given their newly defined subpopulations. $\mathrm{Pd}-1^{+} \mathrm{DN} \mathrm{T}$ cells have been previously described in the spleen and circulation as having an effector phenotype and being the main source of pro-inflammatory cytokines $(24,25)$. However, whether they have a similar phenotype in the kidney, how they contribute to AKI pathology, and through which signaling mechanisms they function, remains unclear. This is of special interest given the great enthusiasm antiPD-1 treatment has received as cancer therapy and raises the question what impact immunosuppressive drugs may have on DN T cell number, activation, and function. Interestingly though, in our hands murine renal DN T cells expressing Pd-1 are rare. Even though their frequency increases with ADPKD, we do not detect them at the same level as published by Sadasivam et al. (16) (Figure 1C). This data may hint towards DN T cells having similar plasticity as other $\mathrm{T}$ cell subtypes where their phenotype, activation, and signaling is susceptible to microenvironmental stimuli. Indeed, Ponzetta et al. recently identified twelve different clusters of tumor-infiltrating DN T cells using singlecell RNA-seq (26), highlighting the potential complexity of these cells. At large, DN T cells have been reported to have suppressive and/or cytotoxic activity towards $\mathrm{CD}^{+}$ and $\mathrm{CD} 8^{+} \mathrm{T}$ cells, B cells and dendritic cells in a Fas/ FasL or perforin/granzyme-dependent manner (27), but, a recent publication showed that DN T cells can also impair the metabolic reprogramming of $\mathrm{CD}^{+} \mathrm{T}$ cells, hence modulating their function (28). Even more interesting, DN T cells have also been reported to be cytotoxic against cancer cells in non-small cell lung cancer (NSCLC) and their numbers increased during treatment with the CTLA4 check-point inhibitor ipilimumab $(29,30)$. Overall, it is clear that the understanding of the pathophysiological function of DN T cells is severely lagging behind their other lymphocyte counterparts.

The lack of knowledge concerning DN T cell function and discrepancies regarding their phenotype or frequency, can at least in part be attributed to the difficulty associated with studying them. By definition, three types of DN T cells exist; Natural Killer-like T (NKT) cells, $\gamma \delta \mathrm{T}$ cells, and "true" DN T cells, all of which express CD3, but are negative for CD4 and CD8. Unfortunately, to date, no exclusive expression markers have been identified to distinguish "true" DN T cells from the other two subtypes, which results in them being defined through exclusion. NKT and "true" DN T cells both express TCR $\alpha \beta$, which allows them to be separated from $\gamma \delta$ T cells, which are TCR $\gamma \delta^{+}$. Further, NKT cells express Nk1.1 in mice and CD56 in human. However, both of these markers are not expressed on all NKT cell types and are not exclusive to that population. A CD1dglycolipid tetramer has been developed to identify all NKT cells, as both NKT type I and II differentiate in the thymus and are positively selected on an MHC I-like, $\beta 2 \mathrm{~m}$-dependent, CD1d molecule (31). This would suggest that DN T cells should be defined as TCR $\alpha \beta^{+}$ CD4 CD 8 CD1 $\mathrm{d}^{-}$as done in Sadasivam et al. (16). However, that definition has not been used in many studies on DN T cells; furthermore, macrophage-like cells expressing TCR $\alpha \beta$ have recently been identified and would be included as DN T cells in this protocol $(32,33)$. Hence, DN T cell frequency as well as their downstream activation status or function, if defined by flow cytometry, can vary drastically dependent on the gating strategy used to identify them (Figure 2). Lastly, it should be noted, that defining a cell type solely by the absence of specific cell surface markers can also be influenced by the digestive protocol used to prepare the single cell suspension for flow cytometry, as antigens can be cleaved off during the experimental preparation of the sample. Additionally, the study of tissue resident DN T cells can be impacted by the presence of circulating DN T cells if extensive perfusion of the organ is not performed prior to organ digestion. All in all, even though complicated to identify, isolate, and study, the current literature suggests that DN T cells likely harbor important functions in physiology and disease. Hence, it is essential to continuously develop new methodologies to study these cells. Only that way will we understand if DN T cells have the potential to be a novel therapeutic target. Studies such as Sadasivam et al. (16), whose group has pioneered the analysis of renal DN T cells, are imperative and advance our understanding of these cells, but additional work is clearly needed. 

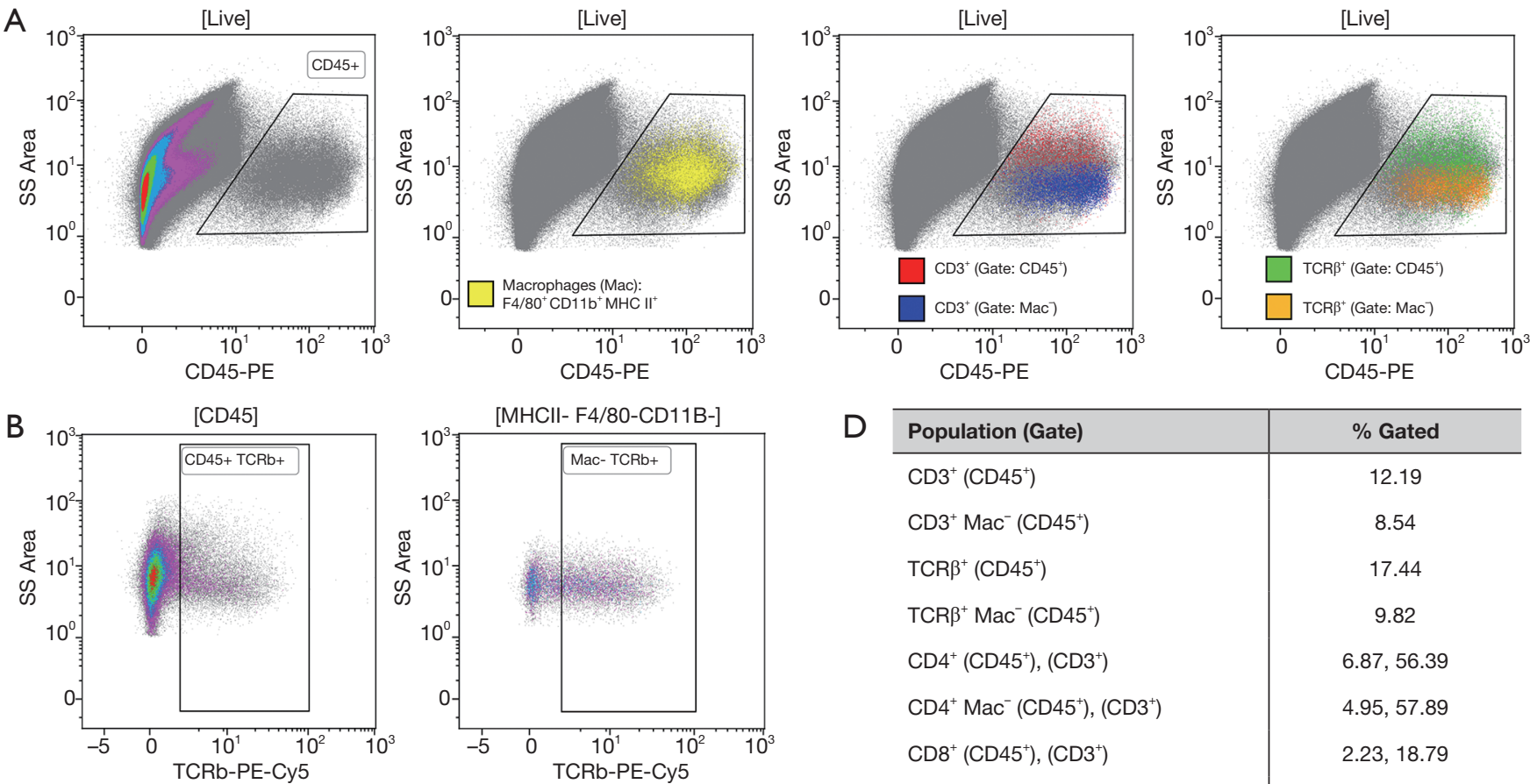

\begin{tabular}{|l|c|}
\hline Population (Gate) & \% Gated \\
\hline $\mathrm{CD}^{+}\left(\mathrm{CD} 45^{+}\right)$ & 12.19 \\
$\mathrm{CD}^{+} \mathrm{Mac}^{-}\left(\mathrm{CD} 45^{+}\right)$ & 8.54 \\
$\mathrm{TCR} \beta^{+}\left(\mathrm{CD} 45^{+}\right)$ & 17.44 \\
$\mathrm{TCR} \beta^{+} \mathrm{Mac}^{-}\left(\mathrm{CD} 45^{+}\right)$ & 9.82
\end{tabular}

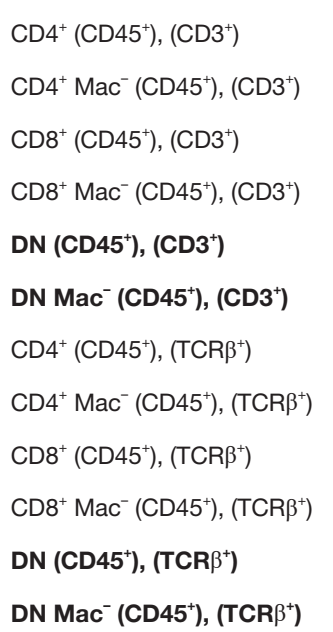

$6.87,56.39$

$4.95,57.89$

$2.23,18.79$

$2.27,27.58$
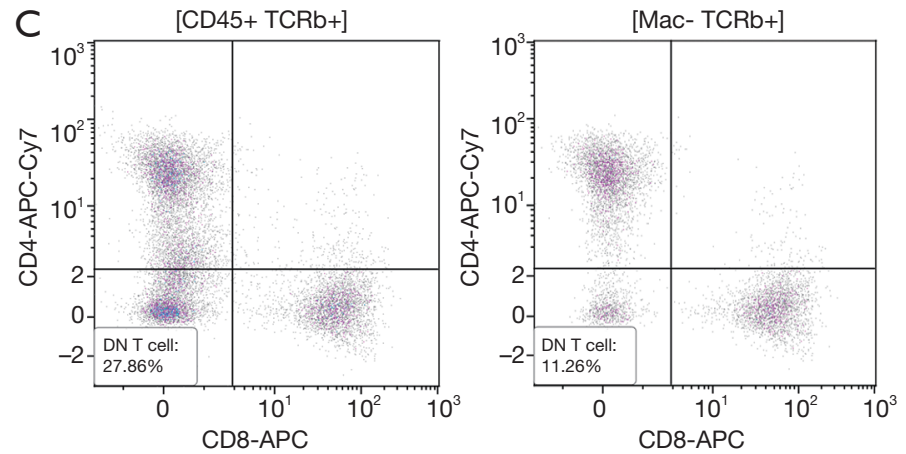

$2.62,21.52$

$1.16,13.61$

$7.97,45.68$

$5.06,51.55$

$4.13,23.71$

$3.45,35.12$

$4.86,27.86$

$1.11,11.26$

Figure 2 Flow cytometry gating strategy can significantly impact DN T cell frequency. Renal single cell suspension of perfused 6-monthold wildtype C57Bl/6 mice were analyzed by flow cytometry. The suspension was first gated on singlets and live cells. (A) The CD45

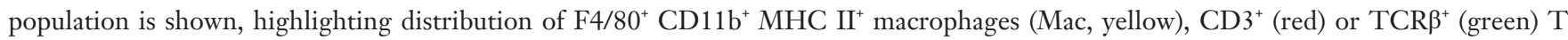
cells if gated directly from the $\mathrm{CD} 5^{+}$population (B, left) and $\mathrm{CD}^{+}$(blue) or $\mathrm{TCR} \beta^{+}$(orange) $\mathrm{T}$ cells if gated after excluding $\mathrm{Macs}$ from the $\mathrm{CD} 45^{+}$population $\left(\mathrm{B}\right.$, right). It is important to note that the $\mathrm{T}$ cell population $\left[\mathrm{CD} 3^{+}\right.$(red) or TCR $\beta^{+}$(green)] significantly overlaps with the Mac population [yellow] if T cells are gated directly of the CD45 gate, supporting the previously published observation that some macrophages are disguised as $\mathrm{T}$ cells $(32,33)$; (C) $\mathrm{CD}^{+} / \mathrm{CD}^{+}$gating diagram highlighting that the frequency of renal $\mathrm{DN} \mathrm{T}$ cells $\left(\mathrm{CD} 4^{-}\right.$ $\mathrm{CD} 8^{-}$) differs significantly dependent on the input gate $\left[\mathrm{CD} 45^{+} \mathrm{TCR} \beta^{+}\right.$(left) or CD $45^{+} \mathrm{TCR} \beta^{+}$excluding Macs (right)]. This suggests that the majority of Macs expressing $\mathrm{T}$ cell receptors are CD4- and CD8 ${ }^{-}$negative and hence could be falsely classified as DN T cells if the wrong gating strategy is applied. Note, our gating protocol is not able to distinguish between "true" DN T cells and NKT cells; (D) tabular summary highlighting how gating can affect renal $\mathrm{CD}^{+}, \mathrm{CD}^{+}$or DN T cell frequency. \% Gated frequencies are an average representative of 6-month-old wildtype C57Bl/6 mice observed using a collagenase I, Liberase LT (Sigma Aldrich) kidney digestion protocol of perfused kidneys as published previously (17). DN, double negative. 


\section{Acknowledgments}

We like to acknowledge the Zell Family Foundation for the support of the University of Colorado, Denver, Anschutz Medical Campus, PKD Program.

Funding: The work was partially supported by a PKD Foundation Research Award and K01DK114164 as well as Pilot \& Feasibility Grants from the Consortium for Fibrosis Research and Translation (University of Colorado, Denver, Anschutz Medical Campus) and the Baltimore PKD Research and Clinical Core Center (University of Maryland); all to K Hopp. EK Kleczko was supported by a T32 (NIDDK 5T32DK007135).

\section{Footnote}

Conflicts of Interest: The authors have no conflicts of interest to declare.

Ethical Statement: The authors are accountable for all aspects of the work in ensuring that questions related to the accuracy or integrity of any part of the work are appropriately investigated and resolved.

\section{References}

1. D'Acquisto F, Crompton T. CD3+CD4-CD8- (double negative) $T$ cells: saviours or villains of the immune response? Biochem Pharmacol 2011;82:333-40.

2. Crispin JC, Oukka M, Bayliss G, et al. Expanded double negative $T$ cells in patients with systemic lupus erythematosus produce IL-17 and infiltrate the kidneys. J Immunol 2008;181:8761-6.

3. Mullauer L, Emhofer J, Wohlfart S, et al. Autoimmune lymphoproliferative syndrome (ALPS) caused by Fas (CD95) mutation mimicking sarcoidosis. Am J Surg Pathol 2008;32:329-34.

4. Marodon G, Warren D, Filomio MC, et al. Productive infection of double-negative T cells with HIV in vivo. Proc Natl Acad Sci U S A 1999;96:11958-63.

5. Cowley SC, Meierovics AI, Frelinger JA, et al. Lung CD4-CD8- double-negative $\mathrm{T}$ cells are prominent producers of IL-17A and IFN-gamma during primary respiratory murine infection with Francisella tularensis live vaccine strain. J Immunol 2010;184:5791-801.

6. Riol-Blanco L, Lazarevic V, Awasthi A, et al. IL-23 receptor regulates unconventional IL-17-producing $\mathrm{T}$ cells that control bacterial infections. J Immunol
2010;184:1710-20.

7. Juvet SC, Zhang L. Double negative regulatory $\mathrm{T}$ cells in transplantation and autoimmunity: recent progress and future directions. J Mol Cell Biol 2012;4:48-58.

8. Duncan B, Nazarov-Stoica C, Surls J, et al. Double negative (CD3+ 4- 8-) TCR alphabeta splenic cells from young NOD mice provide long-lasting protection against type 1 diabetes. PLoS One 2010;5:e11427.

9. Fischer K, Voelkl S, Heymann J, et al. Isolation and characterization of human antigen-specific TCR alpha beta+ CD4(-)CD8- double-negative regulatory $\mathrm{T}$ cells. Blood 2005;105:2828-35.

10. Masuda T, Ohteki T, Abo T, et al. Expansion of the population of double negative CD4-8- $\mathrm{T}$ alpha beta-cells in the liver is a common feature of autoimmune mice. $\mathrm{J}$ Immunol 1991;147:2907-12.

11. Hamad AR. Analysis of gene profile, steady state proliferation and apoptosis of double-negative $\mathrm{T}$ cells in the periphery and gut epithelium provides new insights into the biological functions of the Fas pathway. Immunol Res 2010;47:134-42.

12. Johansson $M$, Lycke $N$. A unique population of extrathymically derived alpha beta TCR+CD4-CD8$T$ cells with regulatory functions dominates the mouse female genital tract. J Immunol 2003;170:1659-66.

13. Ascon DB, Ascon M, Satpute S, et al. Normal mouse kidneys contain activated and CD3+CD4- CD8- doublenegative $\mathrm{T}$ lymphocytes with a distinct TCR repertoire. $\mathrm{J}$ Leukoc Biol 2008;84:1400-9.

14. Martina MN, Noel S, Saxena A, et al. Double-Negative $\alpha \beta$ T Cells Are Early Responders to AKI and Are Found in Human Kidney. J Am Soc Nephrol 2016;27:1113-23.

15. Zimmerman KA, Gonzalez NM, Chumley P, et al. Urinary $\mathrm{T}$ cells correlate with rate of renal function loss in autosomal dominant polycystic kidney disease. Physiol Rep 2019;7:e13951.

16. Sadasivam M, Noel S, Lee SA, et al. Activation and Proliferation of PD-1+ Kidney Double-Negative T Cells Is Dependent on Nonclassical MHC Proteins and IL-2. J Am Soc Nephrol 2019;30:277-92.

17. Kleczko EK, Marsh KH, Tyler LC, et al. CD8+ T cells modulate autosomal dominant polycystic kidney disease progression. Kidney Int 2018;94:1127-40.

18. Hopp K, Ward CJ, Hommerding CJ, et al. Functional polycystin-1 dosage governs autosomal dominant polycystic kidney disease severity. J Clin Invest 2012;122:4257-73.

19. Brandt D, Hedrich CM. TCR $\alpha \beta+C D 3+C D 4-C D 8-$ 
(double negative) $\mathrm{T}$ cells in autoimmunity. Autoimmun Rev 2018;17:422-30.

20. Tapmeier TT, Fearn A, Brown K, et al. Pivotal role of CD4+ T cells in renal fibrosis following ureteric obstruction. Kidney Int 2010;78:351-62.

21. Burne MJ, Daniels F, El Ghandour A, et al. Identification of the CD4(+) $\mathrm{T}$ cell as a major pathogenic factor in ischemic acute renal failure. J Clin Invest 2001;108:1283-90.

22. Baudoux T, Husson C, De Prez E, et al. CD4+ and CD8+ T Cells Exert Regulatory Properties During Experimental Acute Aristolochic Acid Nephropathy. Sci Rep 2018;8:5334.

23. Liu M, Chien CC, Burne-Taney M, et al. A pathophysiologic role for $\mathrm{T}$ lymphocytes in murine acute cisplatin nephrotoxicity. J Am Soc Nephrol 2006;17:765-74.

24. Rodriguez-Rodriguez N, Apostolidis SA, Fitzgerald L, et al. Pro-inflammatory self-reactive $\mathrm{T}$ cells are found within murine TCR- $\alpha \beta(+)$ CD4(-) CD8(-) PD-1(+) cells. Eur J Immunol 2016;46:1383-91.

25. Brandt D, Sergon M, Abraham S, et al. TCR+CD3+CD4CD8- effector $\mathrm{T}$ cells in psoriasis. Clin Immunol 2017;181:51-9.

26. Ponzetta A, Carriero R, Carnevale S, et al. Neutrophils Driving Unconventional T Cells Mediate Resistance against Murine Sarcomas and Selected Human Tumors. Cell 2019;178:346-60.

27. Hillhouse EE, Lesage S. A comprehensive review

Cite this article as: Nemenoff RA, Kleczko EK, Hopp K. Renal double negative T cells: unconventional cells in search of a function. Ann Transl Med 2019;7(Suppl 8):S342. doi: 10.21037/atm.2019.09.107 of the phenotype and function of antigen-specific immunoregulatory double negative T cells. J Autoimmun 2013;40:58-65.

28. Haug T, Aigner M, Peuser MM, et al. Human DoubleNegative Regulatory T-Cells Induce a Metabolic and Functional Switch in Effector T-Cells by Suppressing mTOR Activity. Front Immunol 2019;10:883.

29. Yao J, Ly D, Dervovic D, et al. Human double negative T cells target lung cancer via ligand-dependent mechanisms that can be enhanced by IL-15. J Immunother Cancer 2019;7:17.

30. De Tullio G, Strippoli S, Angarano R, et al. $\alpha \beta$-Double Negative CD4/CD8 (CD56) T cell (DNTs) in metastatic melanoma: basal frequency and behaviour during Ipilimumab treatment. J Transl Med 2015;13:1479-5876.

31. MacDonald HR. CD1d-glycolipid tetramers: A new tool to monitor natural killer $\mathrm{T}$ cells in health and disease. J Exp Med 2000;192:F15-20.

32. Oakley MS, Chorazeczewski JK, Aleshnick M, et al. TCR $\beta$-expressing macrophages induced by a pathogenic murine malaria correlate with parasite burden and enhanced phagocytic activity. PLoS One 2018;13:e0201043.

33. Lee SA, Noel S, Sadasivam M, et al. Characterization of kidney CD45intCD11bintF4/80+MHCII+CX3CR1+Ly 6C- "intermediate mononuclear phagocytic cells". PLoS One 2018;13:e0198608. 